\title{
Electrical properties of rectifying contacts on selectively carrier controlled grown $\mathrm{ZnO}$ thin films
}

\author{
A. Bhattacharya \\ MSU Graduate Student \\ Ram K. Gupta \\ MSU JVIC-CASE \\ Pawan K. Kahol \\ Missouri State University \\ Kartik Ghosh \\ Missouri State University
}

Follow this and additional works at: https://bearworks.missouristate.edu/articles-cnas

\section{Recommended Citation}

Bhattacharya, A., R. K. Gupta, P. K. Kahol, and K. Ghosh. "Electrical properties of rectifying contacts on selectively carrier controlled grown ZnO thin films." Journal of Applied Physics 108, no. 3 (2010): 034514.

This article or document was made available through BearWorks, the institutional repository of Missouri State University. The work contained in it may be protected by copyright and require permission of the copyright holder for reuse or redistribution.

For more information, please contact BearWorks@library.missouristate.edu. 


\title{
Electrical properties of rectifying contacts on selectively carrier controlled grown $\mathrm{ZnO}$ thin films
}

\author{
A. Bhattacharya, R. K. Gupta, P. K. Kahol, and K. Ghosh ${ }^{\text {a) }}$ \\ Department of Physics, Astronomy, and Materials Science, Missouri State University, Springfield, Missouri \\ 65897, USA
}

(Received 23 January 2010; accepted 12 May 2010; published online 10 August 2010)

\begin{abstract}
Controlled decrease in carrier concentration $\left(N_{d}\right)$ through postdeposition annealing of $\mathrm{ZnO}$ is shown to provide a crossover from Ohmic to rectifying junction behavior. Highly oriented (002) $\mathrm{ZnO}$ films with silver contact yield nonlinear $I-V$ characteristics below a carrier concentration of $\sim 10^{23} \mathrm{~m}^{-3}$ and linear Ohmic behavior above $10^{23} \mathrm{~m}^{-3}$. The specific differential resistance around zero bias is practically independent of carrier concentration up to $10^{23} \mathrm{~m}^{-3}$ and then decreases with increase in carrier concentration. These results are in excellent agreement with standard theoretical models of current transport phenomena in metal-semiconductor contacts. While the differential junction resistance at lower carrier concentrations gradually becomes less governed by carrier concentrations and current transport is dominated by thermionic emission and diffusion mechanism, it decreases as a function of carrier concentration at higher carrier concentrations. These results show that metaloxide semiconductor junctions behave precisely like conventional metal-semiconductor junctions.

(C) 2010 American Institute of Physics. [doi:10.1063/1.3447870]
\end{abstract}

\section{INTRODUCTION}

Wide band gap semiconductors have become an attractive candidate in this century, for research, due to their versatile applications. $\mathrm{ZnO}$ is probably the most discussed one in this family for its promising features in the domain of optoelectronics, dilute magnetic semiconductors, and biomedical applications. ${ }^{1-4}$ A large band gap value of $\sim 3.4 \mathrm{eV}$, inherent high charge carrier concentration and high mobility promote it for usage in photodetectors, light emitting diodes, and transparent conducting oxides. ${ }^{5-8}$ Apart from the usage of $\mathrm{ZnO}$ as a transparent conducting electrode in device fabrication, the performance of the end product depends substantially on the nature of the contact between the film and the attached metal.

Based upon growth kinetics and other parameters, the contact could be either of Ohmic or rectifying. Especially the second type of contact, familiar as Schottky contact, forms the basis of many optoelectronics devices. But the formation of thermally stable Schottky barrier remains remarkably challenging even after almost a decade since the very first attempt of Schottky and Mott (1931), to explain the mechanism of barrier formation in metal-semiconductor contact. A number of hurdles to be overcome to form rectifying contact in $\mathrm{ZnO}$, due to natural presence of high charge carrier concentration. This has its root in native defects in $\mathrm{ZnO}$ rising from oxygen vacancies and zinc interstitials. This has therefore, made the limited number of successes in Schottky contacts on $\mathrm{ZnO}$. Some reports draw attention to achieving growth of Schottky barriers between less reactive metals such as $\mathrm{Au}, \mathrm{Ag}, \mathrm{Pd}, \mathrm{Pt}$, and $n-\mathrm{ZnO}$ with barrier height of $0.6-0.8 \mathrm{eV}^{9-13}$ But none of these measurements support the theory of Schottky-Mott. According to Schottky-Mott theory, the effective barrier height $\phi_{\mathrm{B}}$ gets determined by the

${ }^{a)}$ KartikGhosh@missouristate.edu difference between metal work function $\phi_{\mathrm{m}}$ and electron affinity of the semiconductor $\chi_{\mathrm{s}}$. This discrepancy makes way to take into account the effect of defect states originating from vacancies, affecting the formation of barrier. ${ }^{14-18}$ Strong presence of defect states leads to pinning of Fermi level. Also in most of the occasions, the ideality factor $(\eta)$, an important parameter of the junction, stays greater than unity. In recent days, surface treatments methods using chemical etching of $\mathrm{ZnO}$ by $\mathrm{HCl}, \mathrm{H}_{2} \mathrm{O}_{2}$, or oxygen plasma cleaning, are practiced to improve contact properties from Ohmic to rectifying one. ${ }^{19}$ Occasionally, nondestructive treatments like etching through pulsed laser has also shown enhancement of contact performance. ${ }^{20}$ Annealing in oxygen atmosphere, on the other hand, gives a slow but steady control on the quality of the films, in particular, carrier concentration.

In this study, we examine the origin of rectifying behavior in metal-ZnO semiconductor junction and related optoelectronic properties of carrier controlled $\mathrm{ZnO}$ films, which were grown on glass substrate by a pulsed laser deposition (PLD) technique. Being an amorphous substrate, glass is not much used because of difficulties associated with the growth of crystalline films. However, usage of glass makes the overall experiment much more cost effective. It is quite challenging to fabricate oxide films on glass substrate by limiting oxygen vacancies and thus imposing carrier concentration limitations. In general, strong presence of oxygen vacancies leads to the pinning of Fermi level, which does not allow the formation of rectifying contacts. Annealing under oxygen atmosphere offers one methodology in reducing vacancies, present either intrinsically or created due to chemical reactions near the junction. This undoubtedly impacts majority carrier numbers in the conduction band as well as sample resistivity and the carrier mobility. Gradual decrease in oxygen vacancies in the film affected by annealing leads to bet- 
terment in stoichiometry, crystallinity, and more importantly, the carrier concentration, thus setting stage for better and stable rectifying junctions. By gradual decrease in carrier concentration, we have been able to transform an Ohmic junction to a rectifying one. These results are consistent with standard theoretical model of current transport phenomena in metal-semiconductor contacts.

\section{EXPERIMENT}

The $\mathrm{ZnO}$ thin films were grown on glass substrate using PLD (Excel Instrument, Model PLD-STD-19) of sintered $\mathrm{ZnO}$ (99.98\%, Sigma-Aldrich) target. A KrF excimer laser (Lambda Physik, COMPEX, $\lambda=248 \mathrm{~nm}$ and pulsed duration of $20 \mathrm{~ns}$ ) with energy density of $2 \mathrm{~J}_{-} \mathrm{cm}^{-2}$ was used for the deposition. Prior to deposition, the substrates were cleaned in methanol and distilled water by ultrasonic cleaner. The $\mathrm{ZnO}$ films were grown at an optimum temperature of $400{ }^{\circ} \mathrm{C}$ under $\sim 4 \times 10^{-3}$ Torr oxygen pressure. The films were next annealed at $500{ }^{\circ} \mathrm{C}$ and $\sim 2 \times 10^{-2}$ Torr oxygen pressure for different durations of time. The carrier concentration and mobility were calculated from Hall data, collected using standard van der Pauw four probe configuration. Au and Ag electrodes were patterned on top of the $\mathrm{ZnO}$ film by the same PLD technique at $5 \times 10^{-5}$ Torr vacuum. Ag contacts being circular shaped with $300 \mu \mathrm{m}$ diameters were grown at room temperature. Au contacts, the Ohmic one, were grown at $200{ }^{\circ} \mathrm{C}$. The final testing of the device has been performed by $I-V$ characterization, using voltage source and electrometer, products of Keithley Instruments, in combination. Structural parameters of $\mathrm{ZnO}$ films have been calculated from x-ray diffraction (XRD) data collected using x-ray diffractometer (Bruker, Model Discover D8) with source wavelength of $1.5406 \AA$. The root mean square surface roughness of the oxide layer varied between $1-5 \mathrm{~nm}$ over 20 $\times 20 \mu \mathrm{m}^{2}$ as observed by atomic force microscopy (Veeco, Model DI-3100). The UV-Vis spectrophotometer (Ocean Optics, Model HR2000), uses a tungsten-deuterium source, to portray the transmission spectra.

\section{RESULTS AND DISCUSSION}

Figure 1 represents the crystalline orientation of the $\mathrm{ZnO}$ films. The films annealed for different time durations show improvement in terms of sharpness of the (002) oriented peak, which can be attributed to enhancement of surface mobility and lowering of defects. An interesting observation is the peak shift toward higher $2 \theta$ values with change in annealing period. This shift corresponds to contraction of interplanar spacing due to the presence of biaxial stress present in the structure. Annealing under oxygen pressure assists oxygen atoms to relocate at proper lattice sites with the available kinetics. For Williamson-Hall plots ${ }^{21}$ to have a precise view of the nature of strain present in the films, we use the equation:

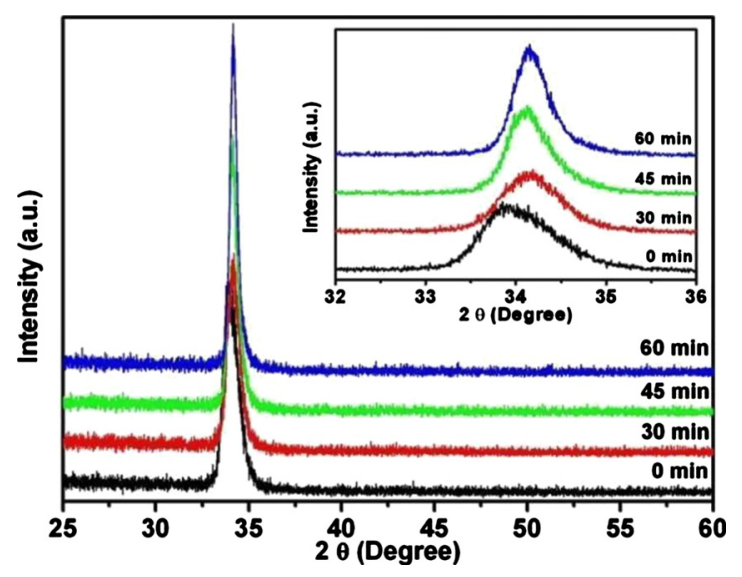

FIG. 1. (Color online) XRD patterns of $\mathrm{ZnO}$ films annealed for different times (inset image show shift in (002) peak of $\mathrm{ZnO}$ films with annealing time).

$$
\beta_{\text {total }}=\beta_{\text {size }}+\beta_{\text {strain }}=\left(\frac{0.9 \lambda}{t} \cos \theta\right)+4\left(\frac{\Delta d}{d}\right)\left(\frac{\sin \theta}{\cos \theta}\right),
$$

where $\beta_{\mathrm{total}}$ is the full width at half maximum, $\lambda$ is the $\mathrm{x}$-ray wavelength, and $\Delta d$ is the change in $d$-spacing of a particular crystal plane. The particle size in our samples varies from $10.4 \mathrm{~nm}$ to $25.5 \mathrm{~nm}$, signifying growth in grain size. The above observations are clearly related to the presence of additional oxygen atoms in the structure.

The film transmittance at different stages of annealing is shown at Fig. 2. The absorption coefficient can be found using the relationship ${ }^{22,23}$

$$
T=A \exp (-\alpha d),
$$

where $T$ is the transmittance, $d$ is the film thickness, and $A$ is a constant close to unity. The optical band gap $E_{g}$ can thus be obtained using the relation

$$
(\alpha h v)^{2}=\left(h v-E_{g}\right) .
$$

In the above equation, $h v$ is the photon energy. The optical band gap is defined as the minimum energy required for

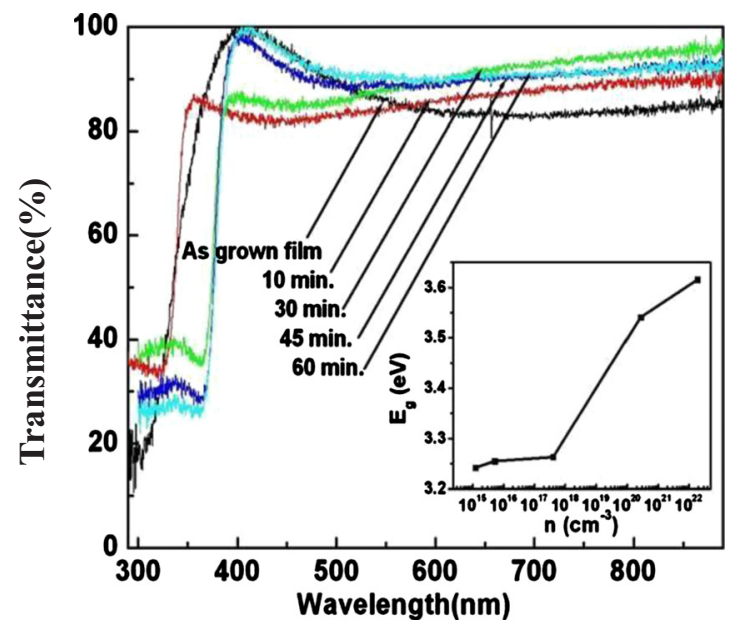

FIG. 2. (Color online) UV-visible spectra of $\mathrm{ZnO}$ films annealed for different times (inset picture shows variation in band gap with carrier concentration). 
an electron to make a transition from valence band to conduction band. An extrapolation of the linear region of the graph $(\alpha h v)^{2}$ with respect to energy $(h v)$ yields the optical band gap, which is found to vary from 3.26 to $3.61 \mathrm{eV}$ with increase in carrier concentration. As-grown film with higher oxygen vacancies shows band gap of nearly $3.61 \mathrm{eV}$. The carrier concentration of as-grown film is found as 3 $\times 10^{26} \mathrm{~m}^{-3}$. At this high carrier concentration, most of the donor electrons occupy states at the bottom of the conduction band which blocks any further low energy optical transitions from taking place. This phenomenon is usually observed for degenerate semiconductors, where the Fermi level lies in the conduction band. The effective band gap, therefore, increases.

The effective expansion of optical band gap, due to restrictions on low energy transitions, is described as BursteinMoss (BM) effect and is given as

$$
E_{e f f}=E_{0}+\Delta E_{\mathrm{BM}},
$$

$E_{0}$, in the above equation, is the band gap of the semiconductor when it is lightly doped. If the conduction and valence bands are assumed to retain parabolic shape, then

$$
\Delta E_{\mathrm{BM}}=\left(\frac{h^{2}}{8 \pi^{2}}\right) /\left(\frac{k_{F}^{2}}{m}\right),
$$

where $k_{F}$ is the Fermi wave number defined as $k_{F}$ $=\left(3 \pi^{2} n_{e}\right)^{1 / 3}$. Some theoretical models, however, take into account the dependence of effective mass on carrier concentration, which leads to nonparabolic bands. ${ }^{24,25}$ In our experiment, we try rigorously to restrict the carrier concentration to less than $10^{23} \mathrm{~m}^{-3}$. We have considered the band gap of lightly doped $\mathrm{ZnO}$ as $3.25 \mathrm{eV}$, as found from our calculation, and have calculated $\Delta E_{g}$ with respect to that. The graph of $\Delta E_{g}$ as a function of $n^{2 / 3}, n$ being the carrier concentration, does not follow linear nature. The deviation may have caused from relaxation of strain present to any particular orientation, which improves the crystallinity of the film, and orients the band gap. As observed, at low carrier concentration, the band gap energy has minor variation.

The large conductivity in defect based oxide semiconductors is due to large donor concentration comes from various defect states such as oxygen vacancies or trivalent impurities such as $\mathrm{Al}, \mathrm{Ga}$, and In. ${ }^{26}$ Donor concentration can be tuned by varying the oxygen pressure during growth or postdeposition annealing under oxygen atmosphere. In Fig. 3, we show the results of our experiments for carrier concentration as a function of annealing time duration. In $\mathrm{ZnO}$ films, electrons are majority carriers. A nascent $\mathrm{ZnO}$ film grown under high vacuum is highly enriched with carriers and its resistivity is very less, which in a few occasions, makes the semiconductor close to a degenerate one. ${ }^{27}$ The resistivity changes appreciably due to annealing. Substantial annealing in oxygen atmosphere raises the resistivity by approximately four orders of magnitude.

The question whether growth of $\mathrm{ZnO}$ films in oxygen and postgrowth annealing treatment have major contributions in plugging defect states depends on structural changes and reactions happening in the film at various stages. Although we do not have a precise answer to this question, we

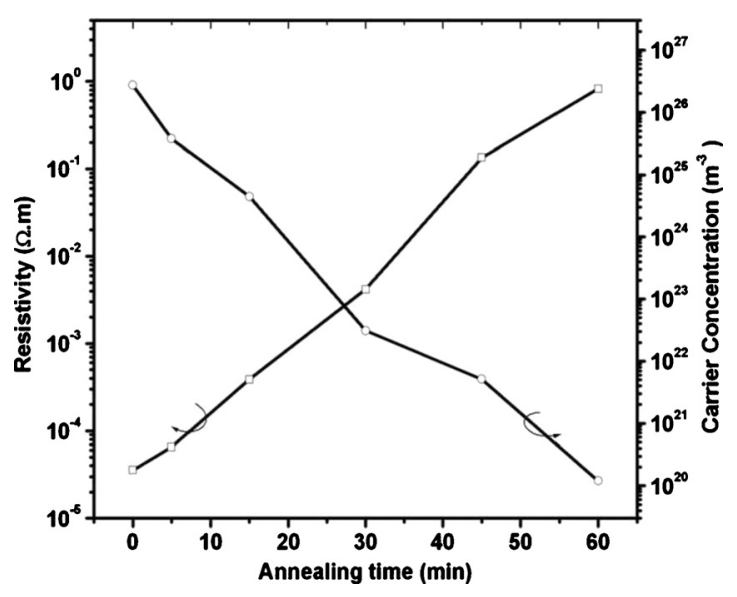

FIG. 3. Variation in resistivity and carrier concentration with postdeposition annealing time.

have tried to look at the junction properties by varying only the oxygen pressure while films were grown. Low oxygen atmosphere $\left(\sim 10^{-3}\right.$ mbar) failed to make much change in the film's nature and, therefore, resulted in Ohmic junctions with relatively low resistance. At slightly higher oxygen pressure $\left(\sim 2 \times 10^{-2}\right.$ mbar $)$, there were a trace of nonlinear $I-V$ behavior. At still higher oxygen content $(\sim 6$ $\times 10^{-2}$ mbar), the films turned out to be highly resistive, making it difficult to observe the nonlinear characteristics. It seems that change in oxygen pressure, abruptly controls oxygen vacancies, making it difficult to gradually observe the transformation of the junction. On the other hand, annealing affects changes steadily and controllably in changing carrier concentration.

In Fig. 4, the $I-V$ characteristics of the junction at different stages of $\mathrm{Ag}-\mathrm{ZnO}-\mathrm{Au}$ configuration are shown. From Figs. 4(a)-4(d), the duration of annealing increases, which influences the carrier concentration. By suitably varying the applied bias and measuring corresponding changes in current, it is observed that the overall characteristics transforms

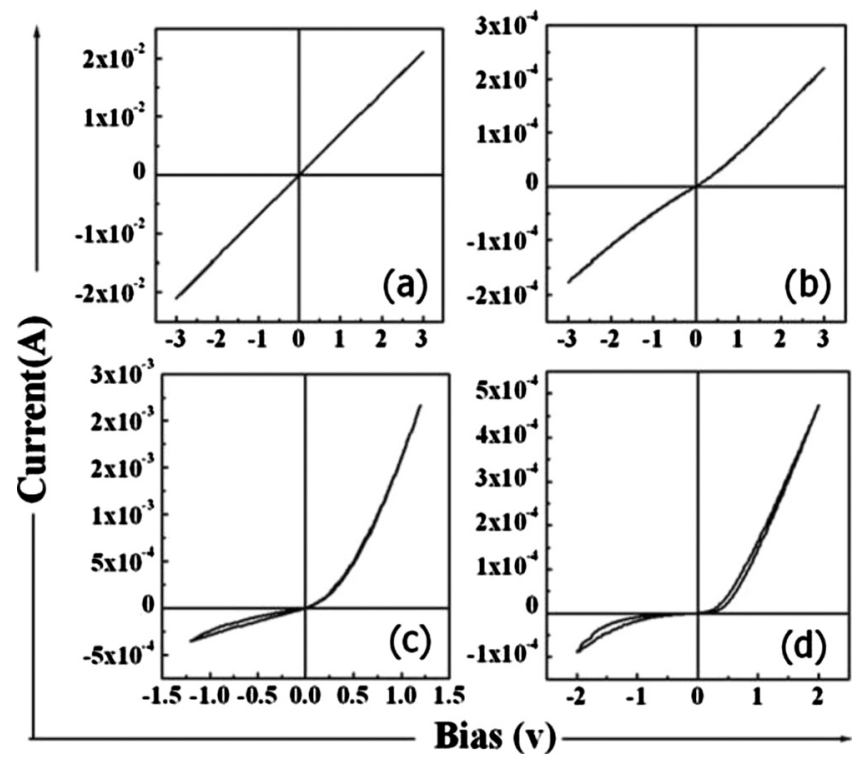

FIG. 4. $I-V$ characteristics of $\mathrm{Ag}-\mathrm{ZnO}$ junction for $\mathrm{ZnO}$ films annealed for (a) $15 \mathrm{~min}$, (b) $30 \mathrm{~min}$, (c) $45 \mathrm{~min}$, and (d) $60 \mathrm{~min}$. 


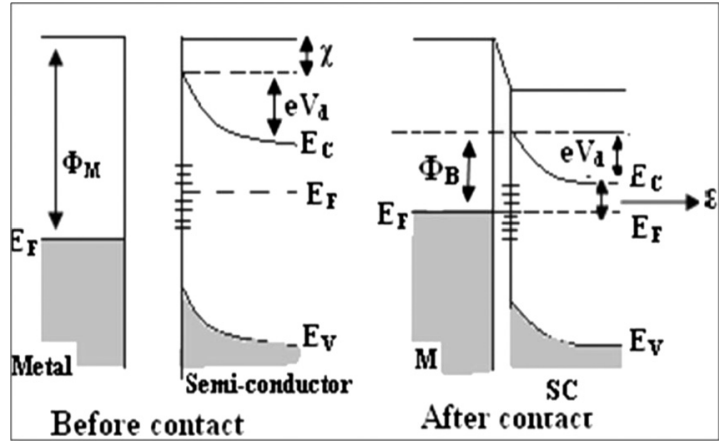

FIG. 5. Contact formation of metal-semiconductor.

from an Ohmic to a rectifying one. Ag has low work function $(\sim 4.3 \mathrm{eV})$. In some occasions it is observed that $\mathrm{Ag}$ forms better thermally stable nonlinear contact with $\mathrm{ZnO}$ (Ref. 28) with high ideality factor due to possible formation of interfacial layer at the junction.

From higher to moderate carrier concentration $\mathrm{Ag}-\mathrm{ZnO}$ contact gradually converts to a stable rectifying contact, whose signature becomes substantially prominent in Figs. 4(c) and 4(d).The reverse saturation current lies around 1.5 $\times 10^{-4} \mathrm{~A}$ at $-0.5 \mathrm{~V}$ for the sample annealed for $45 \mathrm{~min}$, as shown in Fig. 4(c). The reverse saturation current minimizes down to $4.5 \times 10^{-6}$ A for the sample which was annealed for one hour as shown in Fig. 4(d), signifying improvement of reverse saturation current over leakage current in the structure.

To have a deeper understanding of the effect of defect states present in the metal-semiconductor junction, we take the aid of Fig. 5. The defect states which are enriched with electrons provide the surface negative charge prior to metal contacts. The opposite side of the semiconductor therefore becomes positive. The charge density acquired in this region remains uncompensated, thus making the barrier height a function of donor concentration, i.e., on the energies of the defect states and their concentration. ${ }^{29}$ As metal is brought into contact with the semiconductor, Fermi levels of both the materials shift to reach common equilibrium. In this process, charge exchange takes place between the metal and the defect states. This effect of surface states was put forward by Bardeen (1947) (Ref. 30) to remove discrepancy about the dependence of barrier height on metal's work function. $\mathrm{He}$ argued for the presence of a thin insulating layer at the metal-semiconductor interface. The localized states act as source of charges and influence the barrier height. The effective barrier potential here takes the form $\Phi_{B}=\left(e V_{d}+\varepsilon\right)$, where $V_{d}$ is the potential difference across depletion region, $\varepsilon$ is energy difference between Fermi level and conduction band in the semiconductor, and $\chi$ is the electron affinity (Fig. 5). If the number of defect states is sufficiently high, the exchange of charges takes place between surface states and metal, with least effect on the space charges of the semiconductor. In this scenario, the surface states pin the barrier height.

In a forward biased metal-semiconductor junction, electrons can transport in three ways, namely (a) thermionic emission (TE) over the barrier, (b) tunneling or field emis-

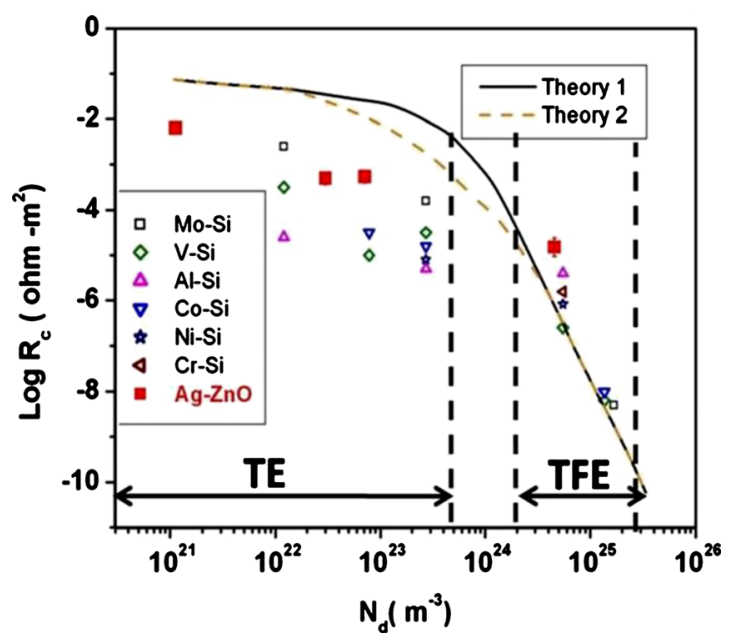

FIG. 6. (Color online) Specific differential resistance $R_{C}$ around zero bias vs doping density $N_{d}$ for various metal contacts to Si (Refs. 32 and 33) and Ag to $\mathrm{ZnO}$ (our data). Dotted and solid curves refer to two different theoretical models (Ref. 34)

sion through the barrier, and (c) carrier recombination and generation in the depletion region. The nature of a junction formed between a metal and a semiconductor may behave as either Ohmic or rectifying depending upon the carrier concentration present in the semiconductor and the transport mechanism. The parameter of paramount importance is the specific differential resistance ${ }^{31} R_{C}=\left.(\delta J / \delta V)^{-1}\right|_{V=0}$. A detailed study by $\mathrm{Yu}^{32}$ showed that $R_{C}$ exhibits three distinct behaviors ${ }^{33} R_{C} \sim \exp \left(\Phi_{b} / E_{00}\right)$, for field emission, i.e., when $q E_{00} / K T \gg 1, R_{C} \sim \exp \left[\Phi_{b} / E_{00} \cosh \left(q E_{00} / K T\right)\right]$, for thermionic field emission (TFE) i.e., when $q E_{00} / K T \sim 1$, and $R_{C}$ $\sim \exp \left(q \phi_{b} / K T\right)$, for TE, i.e., when $q E_{00} / K T \ll 1$.

In the above equations, $E_{00}=(q h / 4 \pi)\left(N_{d} / m^{*} \varepsilon_{s}\right)$, where $m^{*}$ is the electron effective mass and $\varepsilon_{\mathrm{s}}$ is semiconductor's permittivity. For a contact with a semiconductor of high carrier concentration, the conduction is guided by tunneling of thermally excited electrons, described as TFE, where $R_{C}$ is proportional to $\left(N_{d}\right)^{-1 / 2}$. In a semiconductor with moderate or low carrier concentration, the TE takes the charge of conduction in the junction and $R_{C}$ acquires nearly a steady state, independent of $N_{d}$.

Figure 6 shows our results for $R_{C}$ as a function of $N_{d}$ along with the results of two theoretical models and the published results on metal- $n$-type silicon junctions by Hooper, et $a l .{ }^{34}$ The theoretical curves were plotted by Vilms and Wandinger ${ }^{35}$ by using a simple parabolic behavior (dotted line) and a truncated parabolic behavior (continuous curve). Our data on metal- $\mathrm{ZnO}$ contact shows nearly the same trend as the metal-Si contacts show. As evident, below carrier concentration of $10^{23} \mathrm{~m}^{-3}$ the charge transfer is guided by TE.

\section{CONCLUSIONS}

We have made a detailed study of the role of carrier concentration in the formation of Schottky contacts in a metal-ZnO junction. Postdeposition annealing under oxygen atmosphere brings the carrier concentration sufficiently below $10^{23} \mathrm{~m}^{-3}$ to avoid Fermi level pinning. This in turn reduces large contributions from tunneling current, with con- 
comitant increases in resistivity and mobility. Transformation of an Ohmic junction to a rectifying one has been achieved by gradual decrease in carrier concentration. It has been found that nonlinear $I-V$ characteristics of the contacts arise for carrier concentration at and below $10^{23} \mathrm{~m}^{-3}$ and the final barrier voltage is found to lie near $0.6 \mathrm{~V}$. Our results for the $\mathrm{Ag}-\mathrm{ZnO}$ junctions agree with the standard theoretical model of current transport phenomena in conventional metalsemiconductor contacts. At lower doping concentration, the differential junction resistance gradually becomes less governed by carrier concentrations where the current transport is dominated by TE and diffusion mechanism; and at high doping concentration, different resistance becomes function of carrier concentration. In this study, metal-ZnO junction has been shown to be similar to conventional semiconductormetal junctions, this work is believed to further encourage studies on the role of majority carriers and their transport mechanism in observing rectifying junction behavior on wide band gap oxide semiconductors.

\section{ACKNOWLEDGMENTS}

One of the authors (A.B.) would like to acknowledge Dr. S. J. Pearton, Department of Materials Science and Engineering, University of Florida, Gainesville for helpful discussion. Authors are thankful to Mr. Rishi Patel, Centre for Applied Science and Engineering, Missouri State University for collecting AFM data.

${ }^{1}$ S. J. Pearton, D. P. Norton, K. Ip, Y. W. Heo, and T. Steiner, Prog. Mater. Sci. 50, 293 (2005).

${ }^{2}$ M. Nakano, T. Makino, A. Tsukazaki, K. Ueno, A. Ohtomo, T. Fukumura, H. Yuji, S. Akasaka, K. Tamura, K. Nakahara, T. Tanabe, A. Kamisawa, and M. Kawasaki, Appl. Phys. Lett. 93, 123309 (2008).

${ }^{3}$ S. S. Kim, J. H. Moon, B. T. Lee, O. S. Song, and J. H. Je, J. Appl. Phys. 95, 2 (2004).

${ }^{4}$ S. P. Singh, S. K. Arya, P. Pandey, B. D. Malhotra, S. Saha, K. Sreenivas, and V. Gupta, Appl. Phys. Lett. 91, 063901 (2007).

${ }^{5}$ J. Kong, S. Chu, M. Olmedo, L. Li, Z. Yang, and J. Liu, Appl. Phys. Lett. 93, 132113 (2008).

${ }^{6}$ M. Hiramatsu, K. Imaeda, N. Horio, and M. Nawata, J. Vac. Sci. Technol. A 16, 669 (1998).

${ }^{7}$ V. Bhosle, A. Tiwari, and J. Narayan, J. Appl. Phys. 100, 033713 (2006).

${ }^{8}$ W. J. E. Beek, M. M. Wienk, and R. A. J. Janssen, J. Mater. Chem. 15, 2985 (2005).

${ }^{9}$ K. Ip, Y. W. Heo, K. H. Baik, D. P. Norton, S. J. Pearton, S. Kim, J. R. LaRoche, and F. Ren, Appl. Phys. Lett. 84, 15 (2004).

${ }^{10}$ Q. L. Gu, C. K. Cheung, C. C. Ling, A. M. C. Ng, A. B. Djurisic, L. W.
Lu, X. D. Chen, S. Fung, C. D. Beling, and H. C. Ong, J. Appl. Phys. 103, 093706 (2008).

${ }^{11}$ S. Chatman, B. J. Ryan, and K. M. Poduska, Appl. Phys. Lett. 92, 012103 (2008).

${ }^{12}$ B. J. Coppa, R. F. Davis, and R. J. Nemanich, Appl. Phys. Lett. 82, 400 (2003).

${ }^{13}$ M. W. Allen, S. M. Durbin, and J. B. Metson, Appl. Phys. Lett. 91, 053512 (2007).

${ }^{14}$ M. W. Allen and S. M. Durbin, Appl. Phys. Lett. 92, 122110 (2008).

${ }^{15}$ H. L. Mosbacker, Y. M. Strzhemechny, B. D. White, P. E. Smith, D. C. Look, D. C. Reynolds, C. W. Litton, and L. J. Brillson, Appl. Phys. Lett. 87, 012102 (2005).

${ }^{16}$ H. L. Mosbacker, S. El Hage, M. Gonzalez, S. A. Ringelb, M. Hetzer, D. C. Look, G. Cantwell, J. Zhang, J. J. Songd, and L. J. Brillson, J. Vac. Sci. Technol. B 25, 1405 (2007).

${ }^{17}$ L. J. Brillson, H. L. Mosbacker, M. J. Hetzer, Y. Strzhemechny, G. H. Jessen, G. Cantwell, J. Zhang, and J. J. Song, Appl. Phys. Lett. 90, 102116 (2007).

${ }^{18}$ H. L. Mosbacker, C. Zgrabik, M. J. Hetzer, A. Swain, D. C. Look, G. Cantwell, J. Zhang, J. J. Song, and L. J. Brillson, Appl. Phys. Lett. 91, 072102 (2007).

${ }^{19}$ S. H. Kim, H. K. Kim, and T. Y. Seong, Appl. Phys. Lett. 86, 112101 (2005).

${ }^{20}$ M. N. Oh, D. K. Hwang, J. H. Lim, Y. S. Choi, and S. J. Park, Appl. Phys. Lett. 91, 042109 (2007)

${ }^{21}$ J. Badziak, P. Parys, A. B. Vankov, J. Wołowski, and E. Woryna, Appl. Phys. Lett. 79, 21 (2001).

${ }^{22}$ S. T. Tan, B. J. Chen, X. W. Sun, W. J. Fan, H. S. Kwok, X. H. Zhang, and S. J. Chua, J. Appl. Phys. 98, 013505 (2005).

${ }^{23}$ H. C. Pan, M. H. Shiao, C. Y. Su, and C. N. Hsiao, J. Vac. Sci. Technol. A 23, 4 (2005)

${ }^{24}$ B. E. Sernelius, K. F. Berggren, Z. C. Jin, I. Hamberg, and C. G. Granqvist, Phys. Rev. B 37, 10244 (1988).

${ }^{25}$ J. G. Lu, S. Fujita, T. Kawaharamura, H. Nishinaka, Y. Kamada, T. Ohshima, Z. Ye, Y. J. Zeng, Y. Z. Zhang, L. P. Zhu, H. P. He, and B. H. Zhao, J. Appl. Phys. 101, 083705 (2007).

${ }^{26}$ D. C. Look, H. L. Mosbacker, Y. M. Strzhemechny, and L. J. Brillson, Superlattices Microstruct. 38, 406 (2005).

${ }^{27}$ Y. F. Lu, H. Q. Ni, Z. H. Mai, and Z. M. Ren, J. Appl. Phys. 88, 1 (2000).

${ }^{28}$ H. Sheng, S. Muthukumar, N. W. Emanetoglu, and Y. Lu, Appl. Phys. Lett. 80, 12 (2002).

${ }^{29}$ H. K. Henisch, Semiconductor Contacts (Clarendon, Oxford, 1984).

${ }^{30}$ B. L. Sharma, Metal-Semiconductor Schottky Barrier Junctions and Their Applications (Plenum, New York, 1984).

${ }^{31}$ S. M. Sze, Physics of Semiconductor Devices, 2nd ed. (Wiley, New York, 1981).

${ }^{32}$ A. Y. Yu, Solid-State Electron. 13, 239 (1970).

${ }^{33}$ E. H. Rhoderick and R. H. Williams, Metal-Semiconductor Contacts, 2nd ed. (Oxford Science Publications, Oxford, 1988), p. 117.

${ }^{34}$ R. C. Hooper, J. A. Cunningham, and J. G. Harper, Solid-State Electron. 8, 831 (1965).

${ }^{35}$ J. Vilms and L. Wandinger, Ohmic Contacts to Semiconductors (Electrochemical Society, New York, 1969), p. 31. 